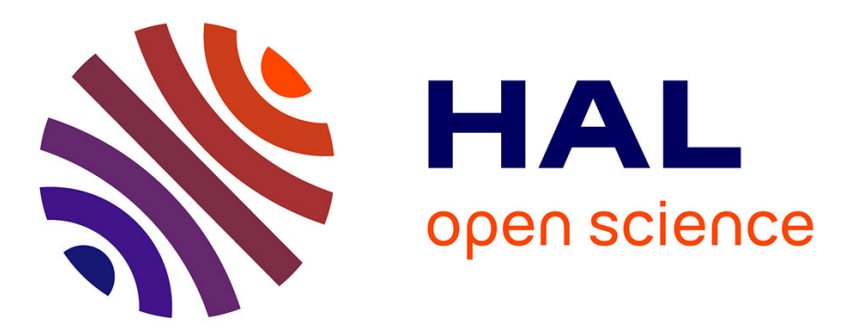

\title{
Rotating spherical Couette flow in a dipolar magnetic field: Experimental study of magneto-inertial waves
}

Denys Schmitt, Thierry Alboussiere, Daniel Brito, Philippe Cardin, Nadège Gagnière, Dominique Jault, Henri-Claude Nataf

\section{- To cite this version:}

Denys Schmitt, Thierry Alboussiere, Daniel Brito, Philippe Cardin, Nadège Gagnière, et al.. Rotating spherical Couette flow in a dipolar magnetic field: Experimental study of magneto-inertial waves. Journal of Fluid Mechanics, 2008, 604, pp.175-197. 10.1017/S0022112008001298 . hal-00264020

\author{
HAL Id: hal-00264020 \\ https://hal.science/hal-00264020
}

Submitted on 13 Mar 2008

HAL is a multi-disciplinary open access archive for the deposit and dissemination of scientific research documents, whether they are published or not. The documents may come from teaching and research institutions in France or abroad, or from public or private research centers.
L'archive ouverte pluridisciplinaire HAL, est destinée au dépôt et à la diffusion de documents scientifiques de niveau recherche, publiés ou non, émanant des établissements d'enseignement et de recherche français ou étrangers, des laboratoires publics ou privés. 


\title{
Rotating spherical Couette flow in a dipolar magnetic field: Experimental study of magneto-inertial waves
}

\author{
By Denys Schmitt, T. Alboussière, D. Brito, P. Cardin, N. Gagnière, \\ D. Jault and H.-C. Nataf \\ Laboratoire de Géophysique Interne et Tectonophysique, CNRS-UJF, BP 53, 38041 Grenoble \\ Cedex 9, France
}

(Received 26 February 2008)

The magnetostrophic regime, in which Lorentz and Coriolis forces are in balance, has been investigated in a rapidly rotating spherical Couette flow experiment. The spherical shell is filled with liquid sodium and permeated by a strong imposed dipolar magnetic field. Azimuthally travelling hydromagnetic waves have been put in evidence through a detailed analysis of electric potential differences measured on the outer sphere, and their properties have been determined. Several types of waves have been identified depending on the relative rotation rates of the inner and outer spheres: they differ by their dispersion relation and by their selection of azimuthal wavenumbers. In addition, these waves constitute the largest contribution to the observed fluctuations, and all of them travel in the retrograde direction in the frame of reference bound to the fluid. We identify these waves as magneto-inertial waves by virtue of the close proximity of the magnetic and inertial characteristic time scales of relevance in our experiment.

\section{Introduction}

Hydromagnetic waves are oscillations propagating in electrically conducting fluids in the presence of a magnetic field. They exist as a result of the different forces acting on a fluid parcel slightly displaced from its equilibrium position. Their characteristics depend on the force balance, in particular on the nature of the leading forces, as well as on the geometry of the container (see a review in Acheson \& Hide, 1973; Finlay, 2005). These forces may be of inertial, buoyancy, magnetic or Coriolis type, two or more of them acting simultaneously. Solving the linearized equations of motion then leads to a dispersion relation $\omega(\mathbf{k})$, the properties of which strongly depend on the force balance and geometry. The simplest case arises when only magnetic (Lorentz) and inertia forces are in competition, giving rise to Alfvén waves of characteristic velocity $v_{A}=B /(\rho \mu)^{1 / 2}$, where $B$ is the amplitude of the magnetic field, $\rho$ the density of the fluid and $\mu$ its magnetic permeability (Alfvén, 1942). When the hydromagnetic system is subjected to a rapid rotation, Coriolis forces may substitute for other ones, at least partially, producing a variety of waves such as inertial, torsional, magneto-Coriolis (MC) or inertial-MC waves, according to the relative magnitude of all forces (Lehnert, 1954b; Braginsky, 1970). Including buoyancy forces adds a further term in the dispersion relation of the so-called MAC (Magnetic, Archimedes, Coriolis) waves (Gubbins \& Roberts, 1987). Considering 
particular boundary conditions, e.g. spherical, may lead to additional restoring forces through specific geometrical constraints ( $\beta$ effect, see Hide, 1966; Malkus, 1967).

From a geophysical point of view, a detailed knowledge of these hydromagnetic waves is fundamental, because they are thought to be present in the Earth's outer core (Braginsky, 1980). One crucial question is whether they might be involved in the secular variation of the Earth's magnetic field (Hide, 1966; Jault et al., 1988; Roberts \& Soward, 1972; Finlay \& Jackson, 2003) or in the occurrence of geomagnetic impulses (Bloxham et al., 2002; Dormy \& Mandea, 2005; Jault \& Légaut, 2005). Another question is whether such hydromagnetic waves are intrinsically stable, or whether they may grow in amplitude through dynamical instabilities (Zhang et al., 2003). Hydromagnetic waves have also been considered in an astrophysical context, because of their possible implication in the redistribution of angular momentum in stellar interiors (Spruit, 1999; Kitchatinov \& Ruediger, 1997; Reese et al., 2004). Similarly, the role of hydromagnetic waves in the destabilization of accretion discs or stellar objects in the presence of differential rotation and toroidal magnetic fields is strongly debated, as this mechanism could lead to the redistribution of angular momentum, hence accretion (Acheson, 1978; Rüdiger et al., 2007).

Few experiments have been performed for studying such hydromagnetic waves in conducting liquids. Pioneering works were carried out from the 50's, initially with mercury (Lundquist, 1949; Motz, 1966). Later, liquid sodium was used, Alfvén waves being generated by mechanical oscillations and analyzed through damping and phase shift measurements (Lehnert, 1954a). In a subsequent piece of work, magnetic excitation was adopted for generating waves in a torus filled with liquid sodium, and a strong resonance was observed, emphasizing the presence of standing Alfvén waves (Jameson, 1964). A revival of interest occurred much later with the quest for experimental dynamos (Stieglitz \& Mueller, 2001; Gailitis et al., 2001). In the successful Karlsruhe dynamo, some oscillations have been attributed to resonant Alfvén waves within the liquid sodium (Mueller et al., 2004). In other experimental devices using liquid metal, spontaneous excitation of oscillating magnetic and velocity fields have been interpreted as the occurrence of magnetorotational instabilities (Sisan et al., 2004; Stefani et al., 2006), while precessing vortices and inertial waves have been identified as well (Sisan et al., 2003; Kelley et al., 2006).

In this paper, experimental results obtained with the "Derviche Tourneur Sodium" (DTS) setup are reported and analyzed. The DTS experiment is a rotating spherical Couette flow device with a strong imposed dipolar magnetic field, and where liquid sodium is used as conducting fluid (Cardin et al., 2002; Nataf et al., 2006). Although it is neither a dynamo experiment nor a reduced model of the Earth core, it has been designed to explore magnetostrophic dynamical regimes similar to those present within the Earth's outer core, its sodium flow being strongly affected by the imposed dipolar magnetic field, although the latter remains far from the complicated field structure expected within the fluid core (Glatzmaier \& Roberts, 1996). Mean axisymmetric properties have been investigated using electric potential and magnetic field measurements as well as ultrasonic Doppler velocimetry (Nataf et al., 2008). Several regimes have been identified as a function of the relative rotation rates of the inner and outer spheres. In particular, a peculiar behaviour has been observed when the inner sphere is rotating in a direction opposite to the outer one, at such a rate that the bulk of the fluid has approximately no absolute rotation. In that case, within a narrow range of differential rotation rates, 
the induced magnetic field is particularly strong. This phenomenon occurs while the radial flow evolves from centripetal to centrifugal in the equatorial plane. The constraint of rotation then vanishes and a strong meridional circulation is allowed, resulting in a strong enhancement of the induced magnetic field.

In the present work, we focus on the time dependence of the electric potential differences between electrodes located on the outer sphere and on the time correlations between those differences. The data have been analyzed in the frequency range [1-50 Hz], i.e. in the same domain as the maximum rotation rate of the spheres $(<30 \mathrm{~Hz})$. Oscillatory patterns have been clearly observed. They are shown to correspond to magneto-inertial waves propagating in a retrograde direction relatively to the core flow. Details on the experimental setup are provided in section 2. The analysis of our data obtained without or with global rotation (i.e. outer sphere at rest or not) is carried out in section 3. Section 4 is devoted to a discussion of the results in order to identify the nature of the waves.

\section{Experimental}

Details of the experimental setup of DTS have been described previously (see Nataf et al., 2006) and are summarized below. Forty liters of liquid sodium are contained between a $7.4 \mathrm{~cm}$ radius inner sphere and a $21 \mathrm{~cm}$ radius outer sphere (Fig. 1). The copper inner sphere is filled with several magnetized bricks producing a nearly dipolar magnetic field within the sodium, the magnitude of which ranges from $0.345 \mathrm{~T}$ to 0.008 $\mathrm{T}$ throughout the volume of fluid. This imposed magnetic dipole is aligned with the rotation axis of the device. The inner and outer spheres can be independently set in rotation around a common axis, their rotation rates being servo-controlled to remain constant. The stainless steel outer sphere is $5 \mathrm{~mm}$ thick, and drilled in several places by $1 \mathrm{~mm}$-diameter, $4 \mathrm{~mm}$-deep holes where electric potential electrodes can be fitted.

We focus here on the results obtained from a set of 18 brass electrodes (labels E1 to E18 on Fig. 1), screwed into some of these holes distributed along two meridians and one parallel. Along both meridians $\left(0^{\circ}\right.$ and $120^{\circ}$ of longitude), electrodes (E1 to E10 and E14 to E18) are placed every $10^{\circ}$ between $-45^{\circ}$ and $+45^{\circ}$ of latitude. The $-45^{\circ}$ latitude parallel electrodes (E14 to E10) are located at $0^{\circ}, 20^{\circ}, 40^{\circ}, 80^{\circ}$ and $120^{\circ}$ of longitude, respectively. The electrodes are close to, but not in direct contact with the sodium flow. However, as stainless steel is a poor electric conductor compared to liquid sodium, it should not affect too much the dynamically generated electric potential. Besides, it is a good enough electric conductor for the impedance of electrode pairs to be much smaller than that of the acquisition system, so that the potential measured by these electrodes is expected to provide a good estimate of the outer boundary potential. Electric signals from the electrodes are passed through slip-rings in the laboratory frame. Electric potential differences are then sampled at a rate of $1000 \mathrm{~Hz}$ with a PXI-6229 National Instruments acquisition card, after going through a simple anti-aliasing $215 \mathrm{~Hz}$ low-pass RC filter. Finally, and to provide additional information, a few measurements of the magnetic field outside the outer sphere are presented, as well as some results of radial flow velocity obtained by ultrasonic Doppler velocimetry. 


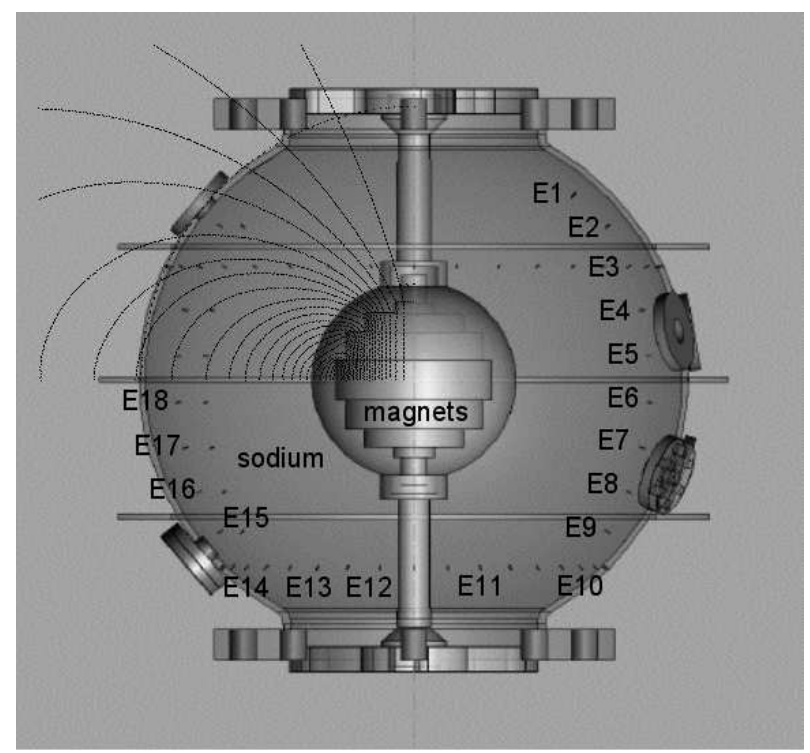

FigURE 1. Sketch of the DTS experiment; the electrodes (labels E1 to E18) are distributed along $0^{\circ}$ and $120^{\circ}$ meridians as well as along the $-45^{\circ}$ parallel between both meridians; magnetic field lines generated by the magnets located inside the inner sphere are also shown.

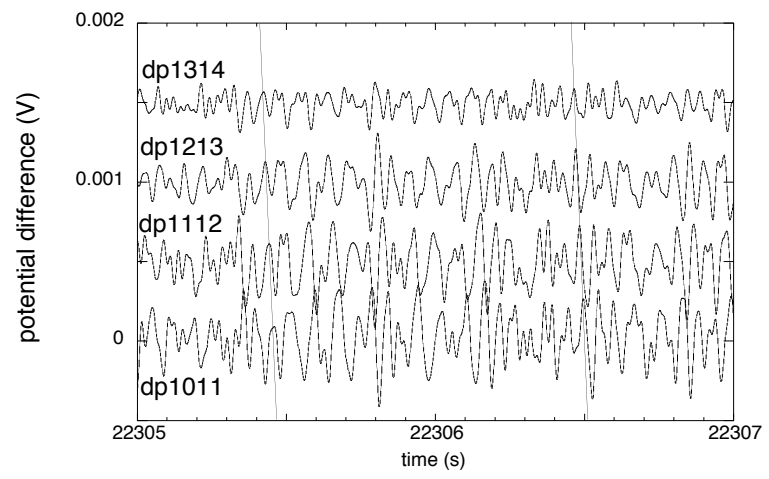

FIGURE 2. Time series of four successive azimuthal potential differences for a differential rotation rate $\Delta f=21.8 \mathrm{~Hz}\left(f_{0}=0\right)$; frequencies above $45 \mathrm{~Hz}$ and around $\Delta f$ have been filtered out; the dashed lines emphasize the relative time shift of the signal with longitude; the data have been arbitrarily shifted in magnitude for the sake of clarity.

\section{Experimental results}

\subsection{Measurements without global rotation}

In this first part, the outer sphere is kept at rest $\left(f_{0}=0\right)$. An example of $2 \mathrm{~s}$ time series for four successive azimuthal DP's (difference of potential), namely dp1011 (between 
electrodes E10 and E11), dp1112, dp1213 and dp1314 is shown on Fig. 2 for a rotation rate of the inner sphere $\Delta f=21.8 \mathrm{~Hz}$. Contrarily to meridional DP's, such an azimuthal DP is not sensitive to the azimuthal mean sodium flow, but to the fluctuations of the meridional circulation, the mean value of which being expected to be very weak (Nataf et al., 2006). Oscillations are clearly visible around a zero averaged value, exhibiting an apparently chaotic behaviour. Nevertheless, a few characteristic frequencies emerge, remaining predominant during short periods of time. Note that the amplitude of dp1011 and dp1112 signals is larger than that of the other ones, in agreement with the larger distance between electrodes. To obtain more details about these frequencies, the PSD (power spectral density) of the electric potential measurements has been calculated. It is shown on Fig. 3 for several differential rotation rates $\Delta f$ varying from 12.6 to $23.4 \mathrm{~Hz}$. These PSD's have been built over a time window of $90 \mathrm{~s}$ within each plateau where $\Delta f$ was kept constant, then averaged over $0.1 \mathrm{~Hz}$. Several narrow peaks are visible, as well as broad structures. The thinnest lines correspond to the rotation rates themselves $\Delta f$ together with their second and third harmonics. Besides, the main feature of these spectra is the presence of broad bumps located at different frequencies, as well as a narrow, distinct peak located at a frequency just higher than $\Delta f$. These structures constitute the major part of the fluctuations. Fig. 3 (bottom) shows temporal magnetic spectra based on the measurement of the radial component $b_{r}$ of the induced magnetic field at latitude $50^{\circ}$ a few millimetres outside the outer sphere. There is a close similarity between the electric and magnetic spectra, at least concerning the broad bumps (in particular, one can observe local maxima around the same frequency values). The observed oscillations have both electric and magnetic signatures.

Spectral density distributions can be visualized with more details using short-time Fourier transform (spectrogram matlab command), in order to observe a possible time evolution of the characteristic frequencies. The corresponding full sequence spectrogram for dp1314 is shown in Fig. 4. Here the constant $\Delta f$ lines and their second harmonics are clearly visible as horizontal segments, while the frequencies of both broad and narrow peaks exhibit a positive slope, i.e. evolve with time. This time dependence can be compared with the variation of meridional DP's for the same sequence of measurement, e.g. dp0910 (i.e. $\Delta V_{-40}$, see Fig. 5). There is indeed a linear relationship between the meridional electric potential difference at a given latitude, say $-40^{\circ}$, and the azimuthal velocity measured by ultrasonic Doppler velocimetry at a certain distance $d$ along the shooting line (see more details in Nataf et al., 2008). This depth $d$ corresponds to a region where the fluid is entrained at an almost uniform angular velocity. Therefore, $\Delta V_{-40}$ can be used as a proxy for the actual angular flow velocity, the coefficient of proportionality being $\gamma \simeq-2700 \mathrm{~Hz} / \mathrm{V}$. From the variation of $\Delta V_{-40}$ over the whole sequence of measurement (Fig. 5), it can then be concluded that the rotation rate of the fluid continues to increase on a very long timescale after a change of $\Delta f$, although $\Delta f$ is maintained constant. This is probably due to variations in the electric coupling between liquid sodium and the copper inner sphere (electrowetting feedback), which gives rise to redistribution of electric currents within the volume, leading then to a different force balance and equilibrium angular velocity. We can take advantage of this variation of the fluid rotation rate to re-examine the frequencies of the oscillations on Fig. 4. The obvious correspondance between both variations emphasizes that the frequency of all these oscillating structures (broad and narrow) is closely linked to the rotation rate of the fluid rather than to that of the inner sphere $\Delta f$.

Deeper knowledge of these oscillations can be gained when the measurements of the 




Figure 3. Power spectral density of dp1011 (top) and of the radial induced magnetic field $b_{r}$ (bottom) for differential rotation rates $\Delta f$ ranging from 12.6 to $23.4 \mathrm{~Hz}\left(f_{0}=0\right)$; triangles indicate the forcing rates $\Delta f$.

successive azimuthal DP's at $-45^{\circ}$ latitude are compared. More precisely the correlation between a pair of distinct DP's, e.g. dp1011 and dp1314, can be analyzed, in order to show a possible propagation of the oscillations along this latitude. However, due to the coexistence of oscillations for several distinct frequencies, it is necessary to filter both signals before computing their cross-correlation. As an example, Fig. 6 shows such a correlation diagram obtained over a time interval of $20 \mathrm{~s}$ within the plateau $\Delta f=18.9$ Hz. The signals have been filtered using a sliding window band-pass filter of width 0.2 Hz. For each analyzed frequency $f$, the amplitude of the cross-correlation as a function of the correlation time is colour-coded. Again, the main structures described above are clearly visible as vertical sequences of alternating red and blue speckles separated by regions with a weak signal (in green). The vertical separation between two successive correlation maxima corresponds to the period of the structure for the selected frequency.

A fundamental feature is that the maximum correlation is shifted from $\delta t=0$ (as for self-correlation) towards a positive value $\delta t_{90}$ of the order of $+0.05 \mathrm{~s}$ (for $f \sim 10 \mathrm{~Hz}$ ). This means that the corresponding oscillating structures are actually moving from (E13E14) to (E10-E11) electrodes, i.e. in the same positive direction as $\Delta f, \delta t_{90}$ being the time spent by the wave to travel between the two pairs of electrodes, distant by about $90^{\circ}$. It has been checked that the time delay $\delta t$ increases linearly with the azimuthal angular distance between the pairs of electrodes considered. Note that this angular 


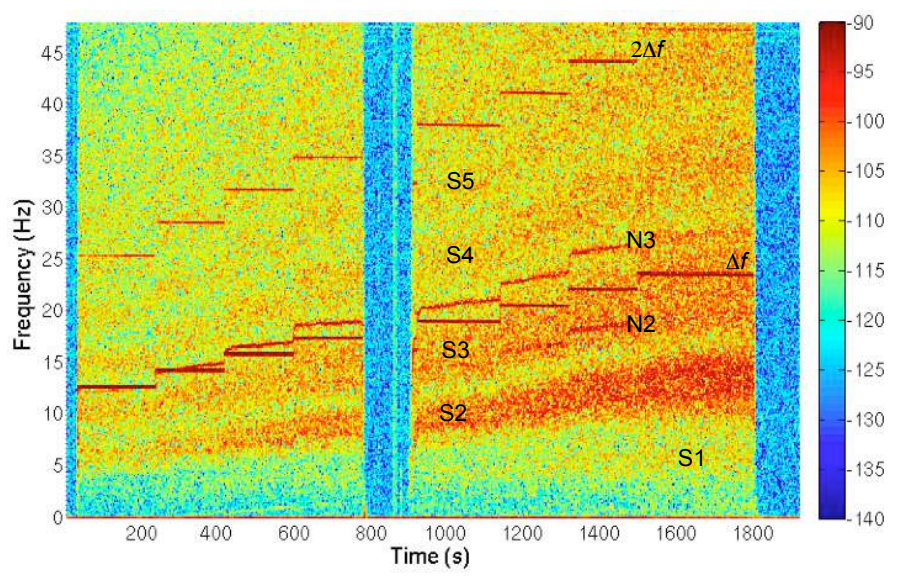

FIGURE 4. Short-time Fourier spectrogram of dp1314 for differential rotation rates $\Delta f$ ranging from 12.6 to $23.4 \mathrm{~Hz}\left(f_{0}=0\right)$; horizontal segments are the traces of $\Delta f$ and $2 \Delta f$, as indicated; labels S2, N3... show the different modes observed, the integer being the azimuthal wavenumber.

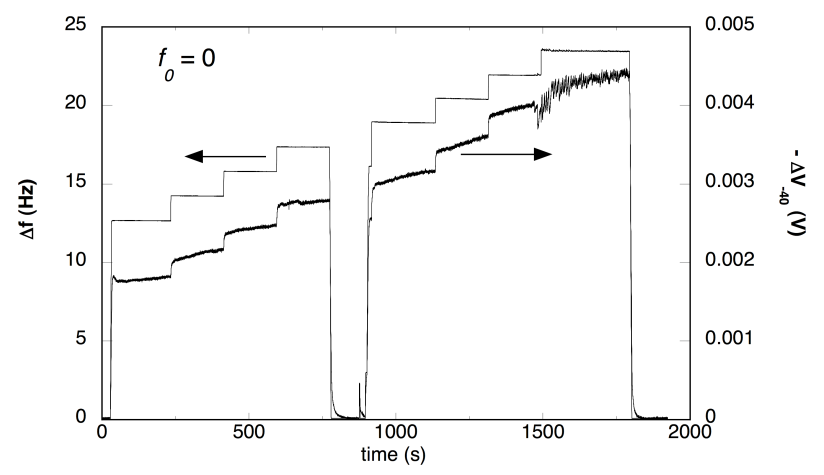

FiguRE 5. Differential rotation rate $\Delta f$ and variation of the $-40^{\circ}$ meridional difference of potential $\Delta V_{-40}\left(f_{0}=0\right)$; note that $\Delta V_{-40}$ can be used as a proxy for the actual averaged rotation rate of the fluid.

dependence of $\delta t$ was already perceptible on the raw data (see Fig. 2), and that a pure transport at the rate of the inner sphere would give $\delta t_{90} \sim 13 \mathrm{~ms}$, far below $50 \mathrm{~ms}$. This characteristic feature demonstrates that the oscillating structures observed in the DTS experiment propagate within the fluid in the same direction as the inner sphere in the reference frame of the outer sphere. This feature is consistent with the existence of hydromagnetic waves.

The knowledge of the time delay $\delta t_{90}$ for a given frequency $f$ allows one to determine an associated wavenumber. Indeed, from the travel time between the two pairs of electrodes, the phase frequency $\tilde{f}=1 /\left(4 \delta t_{90}\right)$ (corresponding to the phase velocity of the wave) can be obtained. This frequency has to be compared with the apparent (measured) frequency $f$. Considering a wave travelling along a parallel with an azimuthal number $m$ and a frequency $f$, i.e. $\mathbf{v}=\mathbf{v}_{\mathbf{0}} \exp i(m \phi-2 \pi f t)$, its phase velocity is given by $2 \pi f / m$, so that the ratio $f / \tilde{f}$ provides the azimuthal wavenumber $m$ of the wave. This change of 


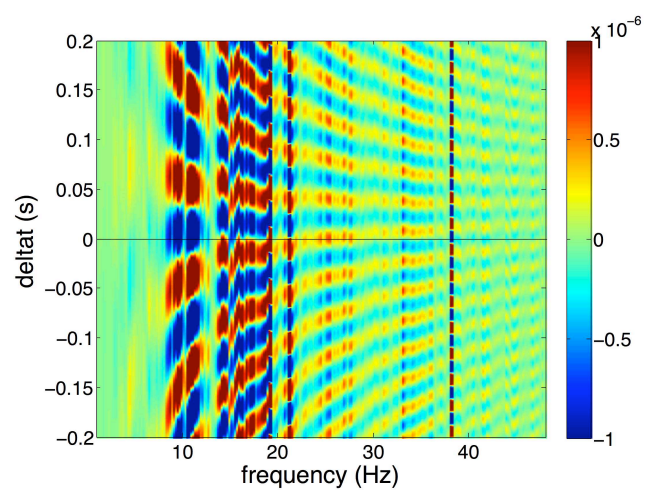

Figure 6. Correlation time - frequency diagram between pairs of difference of potential dp1011 and dp1314 for a rotation rate $\Delta f=$ $18.9 \mathrm{~Hz}\left(f_{0}=0\right)$.

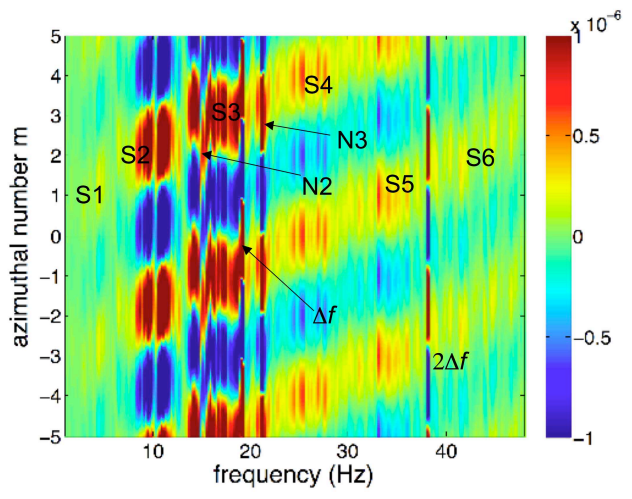

FiguRE 7. Azimuthal number - frequency diagram between both pairs of difference of potential dp1011 and dp1314 for a rotation rate $\Delta f=18.9 \mathrm{~Hz}\left(f_{0}=0\right)$; labels as for Fig. 4 .

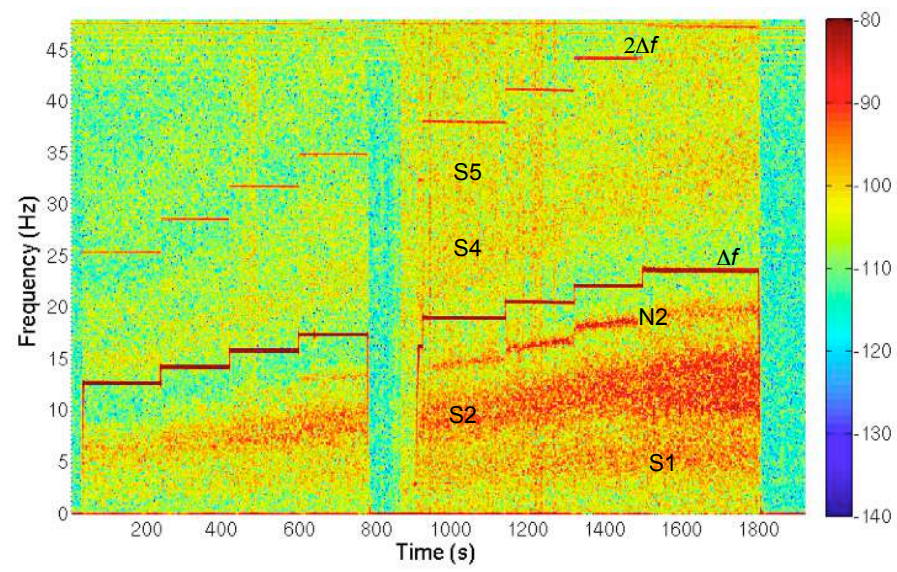

Figure 8. Time-frequency spectrogram of dp1014 for differential rotation rates $\Delta f$ ranging from 12.6 to $23.4 \mathrm{~Hz}\left(f_{0}=0\right)$; note the absence of the $\mathrm{S} 3$ and $\mathrm{N} 3$ waves.

variable from $\delta t_{90}$ to $f / \tilde{f}$ has been repeated for each frequency window, leading to an azimuthal number - frequency diagram as shown on Fig. 7. It becomes obvious now that the successive broad structures seen in the PSD or in the spectrogram shown above (Figs. 3 and 4) may be associated with successive azimuthal wavenumbers $m=2,3$, 4... A fine structure is even perceptible as two or more sub-speckles corresponding to the same wavenumber $m$ but to a different frequency. These sub-structures are generally not separable on the Fourier spectrograms, emphasizing the high resolution of the correlation diagrams. The narrow peak N3 observed at $f=21.1 \mathrm{~Hz}$ is related to a $m=3$ wave. It is clearly distinct from the broad peak S3, which has a lower frequency. A detailed analysis of the diagram reveals another narrow peak N2, related to a $m=2$ wave and located at $f=15.2 \mathrm{~Hz}$, which is again higher than the frequency of the broad peak S2. Finally, $m=1$ oscillations (S1) are also found around $f \sim 5 \mathrm{~Hz}$, although they appear to be extremely weak. 
Pairs of electrodes with different angular spans can provide a different perspective to these wave phenomena. For example, the spectrogram corresponding to the difference of potential dp1014 is shown on Fig. 8. Here E14 and E10 electrodes are separated by $120^{\circ}$, so that any wave with an azimuthal periodicity of $120^{\circ}$ cannot be seen by this DP. Consequently, an extinction phenomenon occurs for the $m=3,6,9 \ldots$ waves (compare with the dp1314 spectrogram in Fig. 4). Conversely, the $m=2$ wave N2 is now clearly revealed above the broad $m=2$ band while it was less perceptible in the broad S3 band on Fig. 4. Studying such extinction phenomena may be useful to emphasize the azimuthal characteristics of the waves present within the fluid, just by selecting an appropriate separation between electrodes.

Now the question arises whether these waves are permanent, and if not, what is their lifetime. To answer this question, a short-time (2 s time window) self-correlation has been performed on dp1314, for a time window sliding along the whole plateau $\Delta f=18.9$ $\mathrm{Hz}$, and after the same sliding window band-pass filtering as described above has been performed. For each pair (time, frequency), the maximum value of the self-correlation function has been considered. All these values have been gathered in a single colour-coded diagram (see Fig. 9). It appears clearly that the waves are far from being permanent, exhibiting a lifetime of a few seconds only. An alternative way for estimating the lifetime of the waves is to consider the envelop of the correlation function calculated over a longer time, the first minimum of the envelop giving the order of magnitude of the mean lifetime of the oscillations. This evaluation provides a similar result, i.e. the lifetime of the waves remains within the range $\sim 2-4 \mathrm{~s}$ (corresponding to 40 to 80 rotation periods). Moreover, these values do not change noticeably when the forcing varies between 0 and $25 \mathrm{~Hz}$, meaning that the attenuation process is magnetic. Finally, note that the different $m$ waves appear independently from each other, suggesting that they are not harmonic components of the same underlying structure.

To summarize the behaviour of the waves observed within the fluid without global rotation $(f=0)$, their frequencies have been extracted from time - frequency correlation diagrams obtained for successive $20 \mathrm{~s}$ time windows along experimental sequences covering differential rotation rates ranging from 0 to $25 \mathrm{~Hz}$. All these values are collected on Fig. 10. All the values have been plotted as a function of $\Delta V_{-40}$, i.e. the meridional $-40^{\circ}$ latitude DP, representative of the actual fluid velocity (see above). As far as the broad bands are concerned, only their mean value has been plotted, while the vertical bars give an estimate of their width. Contrarily to these broad bands which appear at low forcing already, the narrow peaks can be clearly identified only for a rather high rotation rate of the inner sphere. On each plateau of constant $\Delta f$, fluid velocity is not constant (see Fig. 5). When wave frequencies are plotted versus the proxy of fluid velocity, each segment corresponds to a short fraction of the entire curves, following the same trend as the general trend over the whole range (Fig. 10). This behaviour confirms the close link between the wave frequency and the azimuthal fluid velocity. The overall variation appears to be nearly linear, at least for low $m$ azimuthal wavenumbers, while a slight curvature is visible at large $\Delta f$ rate for the highest $m$ values.

\subsection{Measurements with global rotation}

In this second part, the outer sphere is kept at a constant positive rotation rate $f_{0}=$ $4.5 \mathrm{~Hz}$. Starting from the state of solid body rotation $(\Delta f=0)$, the inner sphere can be 


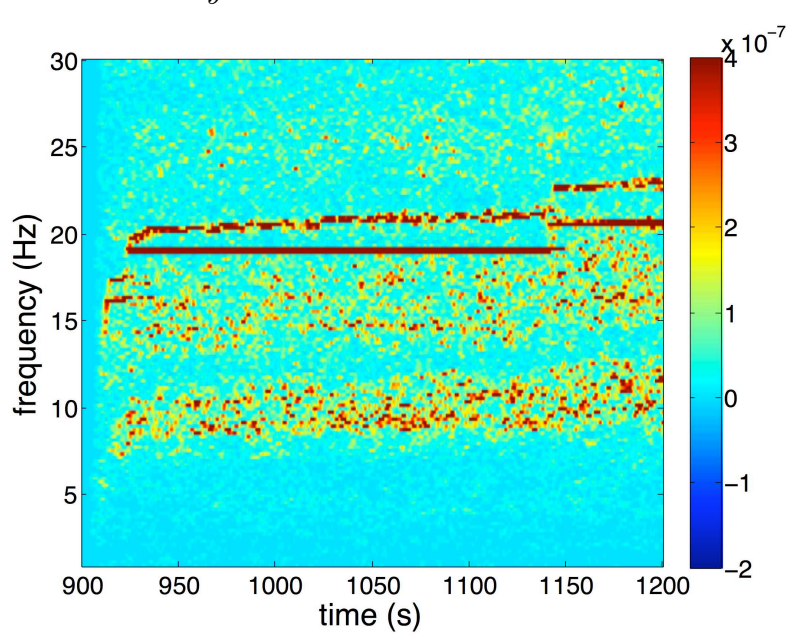

FIGURE 9. Frequency-time diagram of the maximum of dp1314 short-time self-correlation function, corresponding to the plateau $\Delta f=18.9 \mathrm{~Hz}\left(f_{0}=0\right)$.

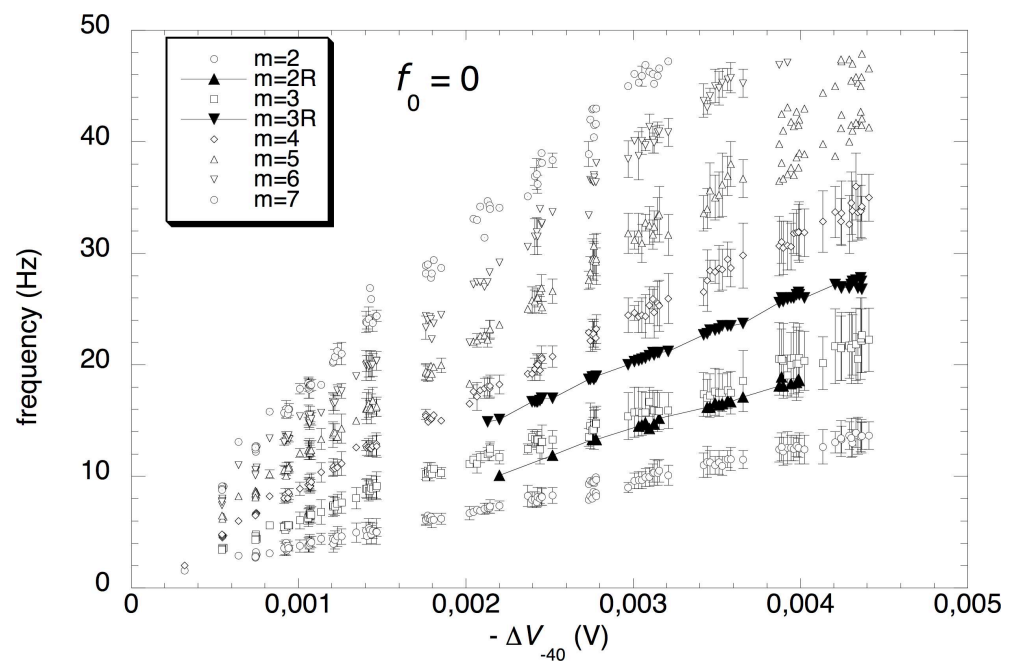

FiguRE 10. Variation of apparent frequencies of the waves as a function of $\Delta V_{-40}$, a proxy of the fluid velocity $\left(f_{0}=0\right)$; for the broad bands, only the mean values have been plotted, and vertical bars correspond to their width.

set to a higher or to a lower rotation rate than $f_{0}$, resulting in a cyclonic or anticyclonic differential rotation, respectively. Note that in the latter case, the forcing can be much more vigourous, since the inner sphere can be ultimately rotated in an opposite direction down to a maximum negative rotation rate $f_{i} \sim-30 \mathrm{~Hz}$, i.e. $\Delta f \sim-35 \mathrm{~Hz}$ (the limit is $\Delta f \sim+25 \mathrm{~Hz}$ in the former case).

\subsubsection{Inner sphere in cyclonic differential rotation}

First, a sequence of DP measurements where $\Delta f$ ranges from 0 to $+18.7 \mathrm{~Hz}$ is considered. As for $f_{0}=0$, power spectral density curves and short-time Fourier spectrograms of azimuthal DP's exhibit oscillatory phenomena, which appear to be much less developed 


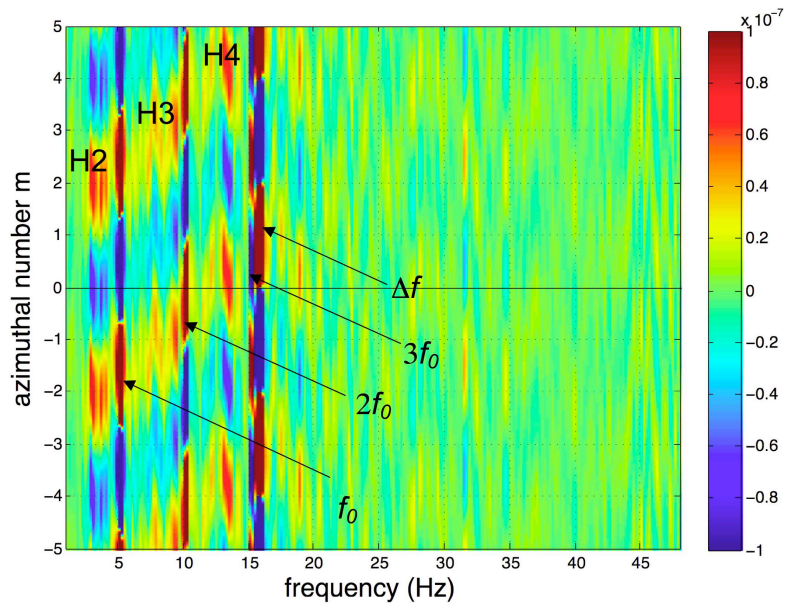

FIGURE 11. Azimuthal wavenumber - frequency correlation diagram between pairs of difference of potential dp1011 and dp1314 for a differential rotation rate $\Delta f=+15.7 \mathrm{~Hz}\left(f_{0}=4.5\right.$ $\mathrm{Hz}$ ); labels H2, H3, H4 show the different modes observed, the integer being the azimuthal wavenumber

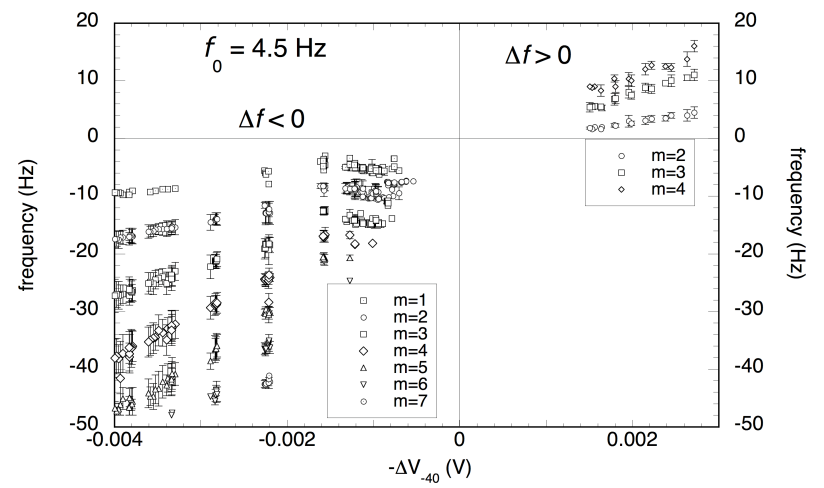

FigURE 12. Variation of apparent frequencies of the waves as a function of $\Delta V_{-40}$, representative of the fluid velocity $\left(f_{0}=4.5 \mathrm{~Hz}\right)$; upper right part: $\Delta f>0$; lower left part: $\Delta f<0$; note that for broad $m$ bands, only their mean frequencies have been reported, and vertical bars correspond to their width.

than without global rotation. First, they start to become apparent only above $\Delta f \sim 10$ Hz. Secondly, they remain confined to a frequency range mainly below $\sim \Delta f$. Thirdly, as shown in the azimuthal wavenumber - frequency correlation diagram between dp1011 and dp1314 at $\Delta f=+15.7 \mathrm{~Hz}$, wavenumbers of hydromagnetic waves are mainly limited to $m=2,3$ and 4 (Fig. 11). Some weak patterns seem to be present above $\Delta f$, but they appear to be much less structured than below $\Delta f$. Finally, all the apparent frequencies of these waves (within a frame rotating with the outer sphere) are gathered on Fig. 12 (upper right part), as a function of the proxy of the fluid velocity relative to the outer sphere, i.e. $\Delta V_{-40}$. 


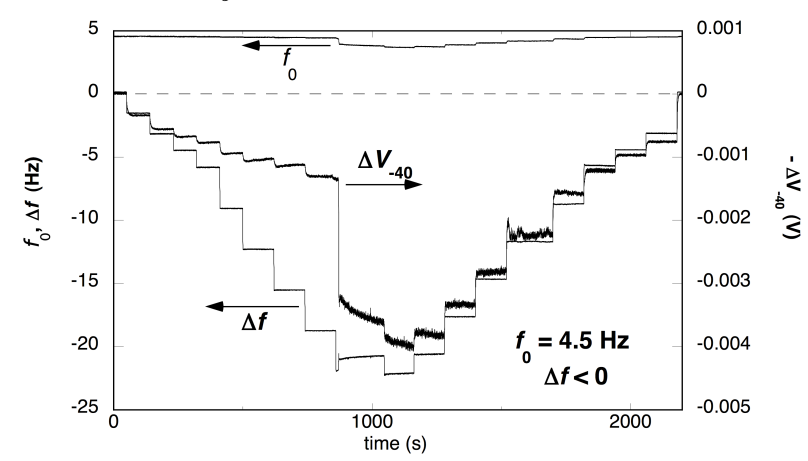

FiguRE 13. Differential rotation rate $\Delta f$ and variation of the $-40^{\circ}$ meridional difference of potential $\Delta V_{-40}\left(f_{0}=4.5 \mathrm{~Hz}, \Delta f<0\right)$; the trace of the outer sphere rotation rate $f_{0}$ is also shown.

\subsubsection{Inner sphere in anticyclonic differential rotation}

We now focus on the experimental results with $f_{0}=+4.5 \mathrm{~Hz}$ and $\Delta f<0$, where a very interesting situation arises when the antagonistic effects of the rotation of both spheres balance and bring most of the fluid to rest. Figure 13 shows such a sequence where $\Delta f$ ranges between 0 and $-22.1 \mathrm{~Hz}$ by successive plateaux where $f_{0}$ is kept constant. This sequence shows a dramatic jump on $\Delta V_{-40}$ when the forcing $\Delta f$ is changed from -18.7 to $-20.8 \mathrm{~Hz}$. This transition separates two distinct regimes for the sodium flow, namely a regime dominated by global rotation and a regime where differential rotation is preponderant (Nataf et al., 2008). The hydromagnetic waves are strongly affected by this transition, and the difference between both regimes is emphasized below.

\section{Weak forcing: a regime dominated by global rotation}

This regime occurs when the forcing is weak, i.e. $|\Delta f|<19 \mathrm{~Hz}(t<860 \mathrm{~s}$ on Fig. 13), which corresponds to a value of $\left|\Delta V_{-40}\right|$ lower than $1.5 \mathrm{mV}$. As described above, a detailed analysis of the cross-correlation between dp1011 and dp1314 DP's associated with two pairs of electrodes distant by $90^{\circ}$ in longitude allows one to identify broad structures corresponding to hydromagnetic waves propagating within the fluid (Fig. 14). By comparison with the spectra observed for $f_{0}=0$, three important differences can be underlined: i) these structures are much less pronounced for quite similar forcing rates; ii) only three frequencies emerge, centered around 5.5, 9 and $14.5 \mathrm{~Hz}$ (labels W1, W2, W3), and no structure is apparent above $15-20 \mathrm{~Hz}$; iii) these characteristic frequencies remain nearly constant (or decrease slightly), while the forcing rate as well as the fluid velocity are increased considerably. In addition to these broad structures, the $f_{0}$ and $\Delta f$ rotation rates, as well as possible second and/or third (and higher) harmonics, are also visible as thin horizontal segments.

In the present weak forcing regime, analyzing the time shift between both DP's indicates that the oscillations are moving westward in the frame of the outer sphere, i.e. in the same negative direction as $\Delta f$. Converting these negative time shifts into azimuthal wavenumbers $m$ by following the same procedure as above leads to the $m-f$ diagram shown on Fig. 15. Here the main speckles (those with the highest correlation function) are associated with a negative $m$ value: they actually correspond to negative wave frequencies and positive $m$ values. Note that if the trace of the forcing rate $\Delta f$ 
is very well marked on these diagrams, the trace relative to the outer sphere rotation rate does not appear so clearly, lying very close to the speckle associated with the $m=1$ fluid oscillation. Nevertheless, $m=1,2$ and 3 waves can be identified without ambiguity, some speckles being possibly subdivided into several components. No indication of waves with $m>3$ seems to be present, nor could one detect the presence of the narrow peaks described in Sec. 3.1 for $f_{0}=0$.

Finally, all the frequencies observed in this weak forcing regime have been collected on Fig. 12. In contrast to the case with no global rotation (Fig. 10), the frequencies appear here to decrease slightly (in magnitude) when the forcing rate is increased. This is a fundamentally different behaviour, which will be discussed later in this paper. Note also that, although the $m=1(2,3)$ wave frequencies are close to the $-f_{0}\left(-2 f_{0},-3 f_{0}\right)$ values, they do not change at the same rate when the forcing is changed, so they cannot be considered to be linked together.

\section{Strong forcing: a regime dominated by shear effects}

We focus now on the strong forcing regime, which arises after the dramatic jump observed on the $\Delta V_{-40}$ variation at $t=870 \mathrm{~s}\left(\left|\Delta V_{-40}\right|>1.5 \mathrm{mV}\right.$, see Fig. 13). Note that, although this transition occurs when the forcing rate changes from -18.7 to $-20.8 \mathrm{~Hz}$, a strong hysteresis is present since a large value of $\Delta V_{-40}$ persists after the forcing rate has been decreased far below $20 \mathrm{~Hz}$ in magnitude. Therefore, although a large value of $\Delta f$ is required to initiate the transition, a high value of $\Delta V_{-40}(>\sim 1.5 \mathrm{mV})$ is actually the relevant criterion to characterize this shear dominated regime (see a discussion in Nataf et al., 2008). Note also that, although the frequency of the outer sphere is expected to remain constant, it actually decreases noticeably after the jump, as the fluid motion becomes faster in the opposite direction, generating a strong antagonistic torque. Nevertheless, this shift remains within the servo-control tolerance for the rotation rate of the spheres.

Analyzing the cross-correlation diagram between dp1011 and dp1314 for this regime of strong forcing reveals a situation fundamentally different from the weak forcing regime (Fig. 14): i) first, a large number of successive bumps is observed in the frequency range 10-50 Hz, corresponding to waves with azimuthal wavenumbers as high as $m=7$; ii) conversely, the $m=1$ signature vanishes almost completely; more precisely the relative proportion $m=1$ vs $m=2$ is completely reversed; iii) the characteristic frequencies of these waves increase again as the forcing becomes stronger. This latter feature is reminiscent of the situation with no global rotation (see Sect. 3.1), emphasizing the predominance of differential over global rotation and the important role played by the strong shear in this regime.

The differences between the rotation dominated and the shear dominated regimes are well emphasized on the $m-f$ diagram of the correlation between dp1011 and dp1314 for the plateau $\Delta f=-17.6 \mathrm{~Hz}$ (Fig. 16). This forcing rate is close to the frequency leading to the spectrogram shown on Fig. 15, as indicated by the position of the thin vertical line associated with $\Delta f$, but the fluid velocity, represented by its proxy $\Delta V_{-40}$, is quite different. While the hydromagnetic waves are confined to $m \leqslant 3$ in the former case, they extend to much higher $m$ values in the latter case. All these frequencies have been gathered on Fig. 12 (left lower part), where both regimes appear to be very distinct from each other. The plateau $\Delta V_{-40}=1.58 \mathrm{mV}(\Delta f=-9.1 \mathrm{~Hz})$ corresponds to an 
intermediate state, which appears to exhibit characteristic features belonging to both regimes: the frequencies of $m \leqslant 3$ waves are consistent with those of the weak forcing regime, while the presence of waves with high $m$ is typical of the regime with strong shear effects. In the latter regime, the variation is similar to the behaviour observed with no global rotation, yet with a slope noticeably steeper by $\sim 10-50 \%$. This will be discussed in Sec. 4.

Ultrasonic Doppler velocimetry does not match electrical potential measurements in terms of spectral resolution (Nataf et al., 2006, 2008). Considering one record nonetheless gives a better understanding of the nature of the waves. Fig. 17 shows the spectrum of radial velocity along a radius as a function of the distance from the ultrasonic probe (fitted on the outer sphere), for an experiment where $f_{0}=4.3 \mathrm{~Hz}$ and $\Delta f=-13.6 \mathrm{~Hz}$. These conditions are actually close to those for $\Delta f=-9 \mathrm{~Hz}$, around $t=1800 \mathrm{~s}$ (Fig. 13), as far as the fluid velocity is concerned $\left(\Delta V_{-40}=1.6 \mathrm{mV}\right.$ in both cases). The waves are observed within the bulk of the fluid under the form of vertical stripes extending between 30 and $90 \mathrm{~mm}$, in the frequency range $5-30 \mathrm{~Hz}$, the most intense ones corresponding to S2 and S3 modes. These waves were first evidenced using azimuthal potential differences, which are thought to be directly related to meridional velocities in the vicinity of the outer sphere. They are also visible on meridional potential measurements (not shown here), which are thought to be directly related to azimuthal velocities in the same region. Now, Doppler velocimetry provides evidence of these waves deep into the volume of liquid sodium. It can be said that these waves are global, not only a phenomenon restricted to a thin external shell close to the outer sphere. Moreover, the radial dependence of the Doppler signature suggests that the waves have low radial wavenumbers, since we do not observe several successive minima as a function of the radius.

\section{Discussion}

In the present spherical Couette experiment, hydromagnetic waves have been evidenced and it has been shown that their characteristic properties depend on whether the flow is dominated by global rotation or by shear effects. In particular, modes of azimuthal wavenumbers $m$ between 1 and 7 have been observed, but their relative magnitude depends on the operating conditions: $m=1$ waves are strong only when the inner sphere is set in weak anticyclonic differential rotation, while $m=2$ modes are dominant in the case of strong shear effects $\left(f_{0}=0\right.$ or $f_{0}>0$ and $\left.\Delta f \ll 0\right)$. These waves exhibit a magnetic signature (except for the N-type waves), they exist at all latitudes and down to a deep level inside the fluid, so that they can be considered as global modes.

Besides, for a given $m$ and a given forcing where the fluid velocity is stationary, the frequency of the waves covers a wide range of values, leading to these broad structures observed in power spectral density curves and in correlation diagrams and exhibiting sometimes sub-structures. One exception is made by the N2 and N3 waves which have been observed only for a high differential rotation rate and no global rotation $\left(f_{0}=0\right)$, and which have much better defined frequencies (narrow spectral peaks).

A crucial point is to identify the actual nature of all these waves and to determine which mechanisms are responsible for triggering and damping them. It can be noticed that the apparent (i.e. measured) frequencies of the modes have been reported in the previous sections as a function of $\Delta V_{-40}$, used as a proxy for the fluid rotation rate 


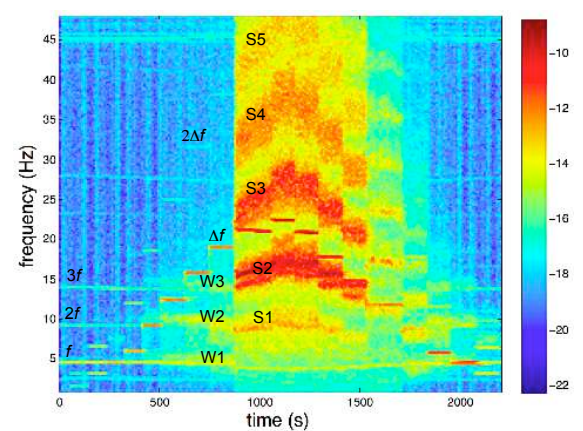

FiguRe 14. Frequency- time diagram of the maximum of dp1011-dp1314 short-time crosscorrelation function, corresponding to the sequence $\Delta f=[0,-22.6 \mathrm{~Hz}]\left(f_{0}=4.5 \mathrm{~Hz}\right)$.

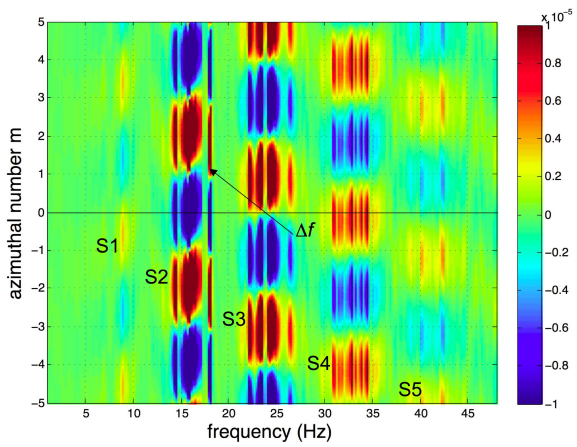

FIGURE 16. Azimuthal number - frequency correlation diagram between pairs of difference of potential dp1011 and dp1314 for a differential rotation rate $\Delta f=-17.6 \mathrm{~Hz}\left(f_{0}=\right.$ $4.5 \mathrm{~Hz}, \Delta V_{-40}=3.3 \mathrm{mV}$ ); note the differences with Fig. 15 with nearly the same forcing.

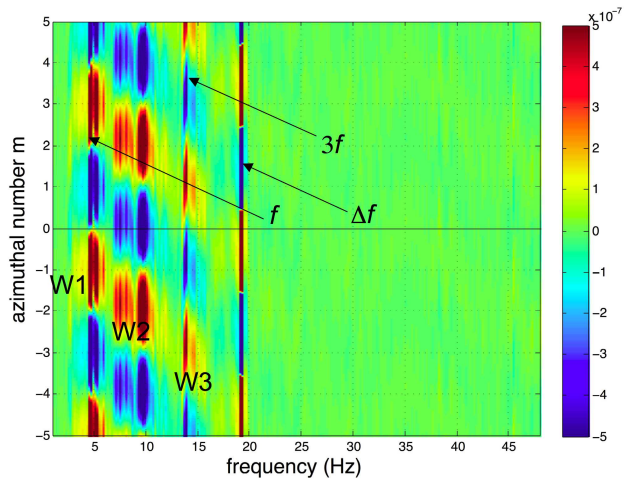

FIGURE 15. Azimuthal number - frequency correlation diagram between pairs of difference of potential dp1011 and dp1314 for a rotation rate $\Delta f=-19.1 \mathrm{~Hz}\left(f_{0}=4.5 \mathrm{~Hz}\right.$, $\left.\Delta V_{-40}=1.3 \mathrm{mV}\right)$

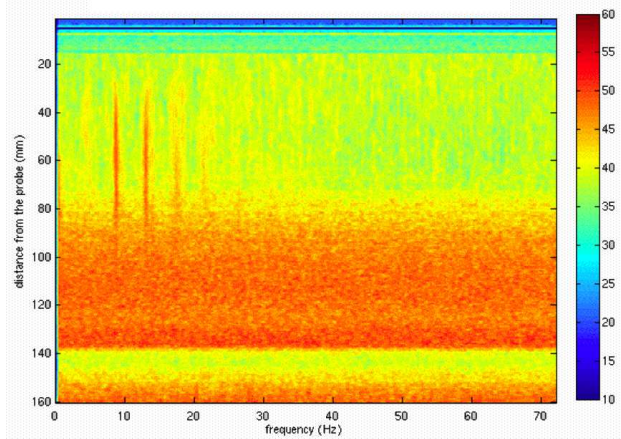

FigURE 17. Color-coded power spectrum of radial velocity in a depth vs frequency plot, obtained by ultrasonic Doppler measurement for a differential rotation rate $\Delta f=-13.6 \mathrm{~Hz}$ $\left(f_{0}=4.3 \mathrm{~Hz}, \Delta V_{-40}=1.7 \mathrm{mV}\right)$; the two main vertical traces are the signature of S2 and S3 modes; note that they occur at a constant frequency down to a noticeable depth (the outer sphere boundary is at the top of the figure, the inner sphere boundary corresponds to a depth of $136 \mathrm{~mm}$ ).

measured within a frame rotating with the outer sphere. However, a more relevant point of view is to analyze the frequencies within a frame rotating with the fluid itself: in this frame, Coriolis forces best represent all inertial effects associated with rotation, and most theoretical studies are based on the analysis of the linear Coriolis forces. As explained in Section 3, and even though the fluid does not rotate at a uniform rate in our experiment, an effective mean rotation rate of the fluid relative to the outer sphere, $f_{\text {fluid }}^{0}$, can be defined as a function of the meridional difference of potential at $-40^{\circ}$ latitude, $\Delta V_{-40}$ :

$$
f_{\text {fluid }}^{0}=\gamma \Delta V_{-40}
$$




$$
=f_{\text {fluid }}-f_{0}
$$

where $f_{\text {fluid }}$ and $f_{0}$ are the fluid and outer sphere rotation rates, respectively, within the laboratory frame, and where $\gamma$ has been estimated to be $\simeq-2700 \mathrm{~Hz} / \mathrm{V}$ from ultrasonic Doppler measurements. If we consider a wave propagating along a parallel with an azimuthal number $m$ and a frequency $f_{\text {mode }}^{0}$ in the frame of the outer sphere, e.g. $\mathbf{v}=\mathbf{v}_{\mathbf{0}}$ $\exp i\left(m \phi-2 \pi f_{\text {mode }}^{0} t\right)$, its frequency is Doppler shifted in the frame of the rotating fluid, according to:

$$
\begin{aligned}
f_{\text {mode }} & =f_{\text {mode }}^{0}-m f_{\text {fluid }}^{0} \\
& =f_{\text {mode }}^{0}-m \gamma \Delta V_{-40}
\end{aligned}
$$

In Fig. 18 we have gathered the wave frequencies $f_{\text {mode }}$ as a function of the effective rotation rate of the fluid in the laboratory frame, i.e. $f_{\text {fluid }}$, for $\gamma=-2700 \mathrm{~Hz} / \mathrm{V}$, for the three sets of measurements shown above. Focusing on the case $f_{0}=4.5 \mathrm{~Hz}, \Delta f<0$, the point $\left(f_{\text {fluid }}=0, f_{\text {mode }}=0\right)$ emerges as a singular point of convergence for all curves, even for the $\mathrm{W}$-type waves whose frequency was nearly constant in the frame of the outer sphere. This peculiar point corresponds to the situation where the fluid is at rest, on average, in the laboratory frame. It is worth emphasizing that at this point, the induced magnetic field is strongly enhanced (Nataf et al., 2008) and that the wave frequencies vanish. The latter property has been directly observed by the magnetic sensors in the laboratory frame, as oscillations showing very long periods ( 10-20 s) were recorded. In this representation (Fig. 18), some dispersion and deviations may occur, emphasizing the limit of the approximation given in Eq. 4.1. Note that with this value of $\gamma$, the fluid may be entrained by the inner sphere at a rate up to half the differential rotation rate, as a result of the strong magnetic coupling between the permanent magnets located inside the inner sphere and the conducting fluid.

Considering now all these experimental results $f_{\text {mode }}$ (in the frame of the fluid) as a function of $f_{\text {fluid }}$ (in the frame of the laboratory) allows one to identify four distinct types of waves, all retrograde, and all exhibiting a roughly linear dependence of frequency on the inertial rotation rate of the fluid:

(a) Waves of type S: they cover a wide range of fluid rotation rate $-8<f_{\text {fluid }}<14$ $\mathrm{Hz}$, including the region with a weak rotation rate; they are roughly symmetric with respect to the origin; all the modes $1 \leqslant m \leqslant 7$ have been observed, although the $m=$ 1 modes are much weaker than the other ones; they exist in shear-dominated regimes, with or without global rotation $\left(|\Delta f|>f_{0}=0\right.$, or $\left.\Delta f / f_{0} \ll-1\right)$;

(b) Waves of type $\mathrm{N}$ : they correspond to the narrow structures in the spectrograms; only $m=2$ and 3 modes have been observed; they have been seen for $f_{0}=0$ only, although not systematically; they are slower than the S-waves;

(c) Waves of type $\mathrm{H}$ : they are observed for a high cyclonic forcing, e.g. $\Delta f / f_{0} \gg 1$; their frequency is similar to that of S-waves although only $2 \leqslant m \leqslant 4$ modes have been observed; they exist in regimes dominated by both shear and rotation;

(d) Waves of type W: they are present when the fluid is rotating more slowly than the outer sphere, in the same direction, i.e. a weak anticyclonic forcing regime, e.g. $\Delta f<0<f_{\text {fluid }}<f_{0}$; only $m \leqslant 3$ modes have been observed; they are noticeably faster 

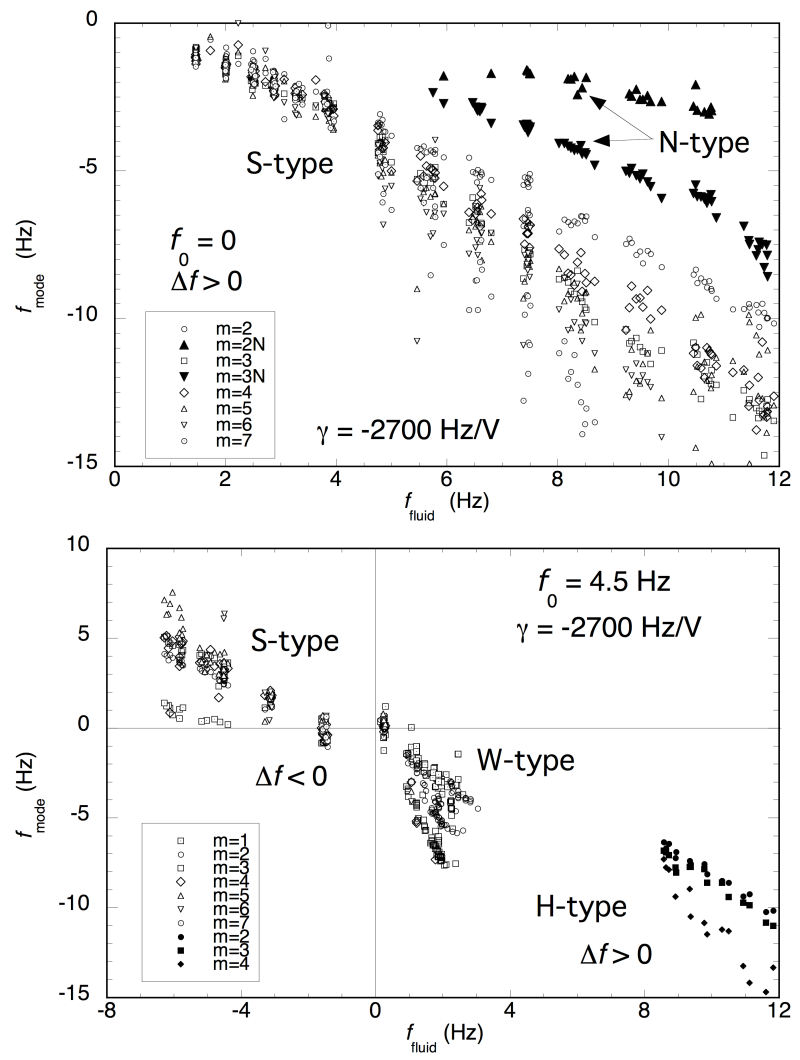

FigURE 18. Variation of the wave frequencies relative to the fluid as a function of the effective fluid rotation rate $f_{\text {fluid }}$ in the laboratory frame $(\gamma=-2700 \mathrm{~Hz} / \mathrm{V})$; upper part: $f_{0}=0, \Delta f>0$; lower part: $f_{0}>0, \Delta f>0$ or $<0$.

than the S-waves; they exist in weak forcing, rotation-dominated regimes; note also that this regime is the only one where the angular velocity of the fluid increases outwards.

Dispersion relations can be built from the above variations, as a function of the $m$ wavenumber (Fig. 19). In order to compare these relations for the different types of waves, the frequencies have been renormalized by the fluid velocity. Moreover, only an averaged value is reported for each $m$ wavenumber, the approximation used in our analysis limiting the accuracy of the numerical values. The dispersion relations for the S-type waves appear very similar for both configurations $\Delta f>f_{0}=0$ and $\Delta f / f_{0} \ll-1$, and close to the variation for the H-type waves. So, both types of waves could be of the same nature, but the absence of high $m$ values for the $\mathrm{H}$-waves remains to be explained. The other types of waves ( $\mathrm{N}$ and $\mathrm{W}$ ) are clearly different, with variations outside the range of the previous ones.

Few theoretical studies have been devoted to the existence of hydromagnetic oscillations in rotating conducting fluids. In the spherical geometry, oscillations have been first investigated in thin and thick spherical shells (Hide, 1966), but from a local point of view where Coriolis forces depend on latitude ( $\beta$-plane model). Two solutions emerge from this model, within the assumption of large wavelengths: the first one is a Rossbytype, low-frequency inertial wave, while the second one is a MC- (magneto-Coriolis) 


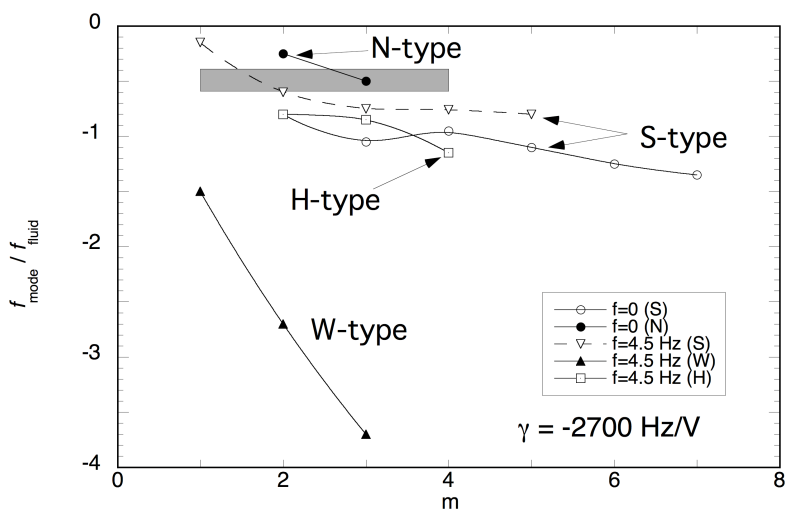

FigURE 19. Dispersion relation of the four types of waves; only averaged values are reported; grey rectangle: region where inertial waves have been observed by Kelley et al. (2007).

wave, resulting from the balance between Lorentz (magnetic) and latitudinally varying Coriolis forces. However, for both types of waves, characteristic frequencies are strongly dependent on latitude, a variation not observed in the present measurements.

Later, the full spherical geometry has been considered (Malkus, 1967), a situation closer to our experimental configuration. In this model, where a toroidal magnetic field with a magnitude proportional to the distance to the rotation axis is considered, two solutions emerge again from the wave equations within the frame of the rotating fluid. In the case of large wavelengths (small $m$ ), solutions reduce to pure inertial-type waves, and pure Alfvén-type MC-waves, but in the general case, both solutions are mixed. The present results (Fig. 18) appear to be more consistent with inertial-type waves, at least for the S-, N- and possibly $\mathrm{H}$-type waves (linear dependence on $f_{\text {fluid }},\left|f_{\text {mode }}\right|$ values smaller than $2 f_{\text {fluid }}$ ). However, their typical frequencies imply a high polar wavenumber $n$, which is not seen in our data using Doppler velocimetry. In contrast, W-type waves cannot be pure inertial-type modes, since their frequencies exceed this $2 f_{\text {fluid }}$ limit. Nevertheless, an Alfvén-type MC-wave solution cannot be excluded, but going further in the analysis would require to know more precisely the geometry and magnitude of the induced toroidal magnetic field.

Let us now compare our results with those obtained recently in other liquid sodium experiments. In both Riga (Gailitis et al., 2003) and VKS (Monchaux et al., 2007) dynamo experiments, the possible role of hydromagnetic waves for dynamo generation has not been invoked. In the Karlsruhe dynamo experiment, oscillations have been observed on the power spectral density of the induced magnetic field, in a way reminiscent of our results, but it was within a kinematic dynamo regime and the geometry was far from the DTS one (Mueller et al., 2004). Their typical frequencies (in the range $0.7-3 \mathrm{~Hz}$ ) agree well with the reciprocal transit times of the Alfvén waves along the characteristic structural length scale of their experimental device, namely two diameters of a vortex generator. These oscillations have been suggested to reflect a resonant interaction of Alfvén waves with these structural length scales, with the additional hypothesis of the existence of a mechanism able to sustain the waves, which should be otherwise strongly damped in liquid sodium on length scales of about $0.1 \mathrm{~m}$. In contrast, in the DTS experiment, the waves cannot be pure Alfvén waves because of the presence of the strong rotation. Moreover, Alfvén waves should be expected to be the fastest in the vicinity of 


\begin{tabular}{|c|c|c|c|c|}
\hline symbol & name & definition & value & unit \\
\hline$a$ & outer radius & & 0.21 & $\mathrm{~m}$ \\
$b$ & inner radius & & 0.074 & $\mathrm{~m}$ \\
$\eta$ & magnetic diffusivity & & 0.087 & $\mathrm{~m}^{2} / \mathrm{s}$ \\
$P m$ & magnetic Prandtl number & $\nu / \eta$ & $7.510^{-6}$ & \\
$B_{0}$ & imposed magnetic field & & 0.008 & $\mathrm{~T}$ \\
\hline$R m$ & magnetic Reynolds number & $2 \pi a b \Delta f / \eta$ & $\sim 20$. & \\
$H a$ & Hartman number & $a B_{0} / \sqrt{\rho \mu \nu \eta}$ & $\sim 200$. & \\
$B_{\text {tor }}$ & induced magnetic field & $\sim R m B_{0}$ & $\sim 0.2$ & $\mathrm{~T}$ \\
$v_{A}$ & Alfvén velocity & $B_{\text {tor }} / \sqrt{\rho \mu}$ & $\sim 5$. & $\mathrm{m} / \mathrm{s}$ \\
$S$ & Lundquist number & $a v_{A} / \eta$ & $\sim 10$. & \\
\hline
\end{tabular}

TABLE 1. A few parameters and indicative values of dimensionless numbers relevant for this work; the differential rotation rate of the inner sphere has been taken as $\Delta f=20 \mathrm{~Hz} ; B_{0}$ is given at the outer sphere equator; an order of magnitude for the induced toroidal magnetic field $B_{\text {tor }}$ is also given.

the singular point $\left(f_{\text {fluid }} \sim 0\right)$ where the induced meridional magnetic field exhibits a strong peak, while our waves show vanishing frequencies. Concerning the life-time of the waves (a few seconds), the Lundquist number $S$ has to be considered, since a condition for Alfvén waves (or any waves linked to the induced magnetic field) to be detected is $S \gg 1$ (Roberts, 1967). This parameter characterizes the ratio between the ohmic damping time scale and the typical period of the wave. In DTS, $S$ can reach a value as high as $\sim 10$, a value rather large for an experimental device (see Table 1), leading to characteristic time scales of a few seconds for waves with frequencies of about $10 \mathrm{~Hz}$.

Much closer to our configuration is another spherical Couette flow experiment where instabilities have been observed from magnetic measurements outside the fluid, and interpreted as magnetorotational (MRI) instabilities (Sisan et al., 2004). There, dominant $m=1$ oscillations have been detected, with characteristic frequencies around $30-50 \%$ of the rotating rate of the fluid (the outer sphere was at rest). Moreover, they have been identified in a limited domain of the possible values of homogeneous external magnetic field and differential rotation strengths. In our data, we did not observe such a phase diagram as a function of the forcing, although our characteristic frequencies are similar. A different configuration has been also investigated, with two corotating impellers instead of a rotating inner sphere, but with the same spherical outer sphere and the same homogeneous magnetic field, parallel to the rotation axis (Sisan et al., 2003). Instabilities (only $m=1$ and 2) were also observed for certain ranges of applied magnetic field. These oscillations have been interpreted as precessing columnar vortices aligned with the magnetic field. Their frequencies are higher than the MRI frequencies quoted above, and closer to our typical values. However, the absence of correlation between different $m$ components in our results is not in favour of the presence of coherent vortices in DTS.

More recently, the same spherical Couette flow geometry has been investigated, with two rotating concentric spheres larger than in the above experiment, and again a constant imposed magnetic field parallel to the rotation axis (Kelley et al., 2007). The obtained spectrograms exhibit some similarities with our results. In particular, $m=1,2$ and 3 oscillating structures have been identified in their induced magnetic field data. Based on an array of 21 meridional Hall probes mounted in the non-rotating frame, a full map of the structure of these patterns could be built. From an analysis of their geometry 
and frequency, they have been interpreted as being inertial modes excited by strong differential rotation at the cylindrical surface tangent to the inner sphere. Broader, more diffuse structures were also visible on their spectrograms, in a way reminiscent of our S-type waves. However, the dependence of the observed frequencies on the differential rotation and on azimuthal wavenumber $m$ generally differs from the variations shown in the present work (see Fig. 19). The inertial waves identified by Kelley et al. (2007) have been associated with high power bands, i.e. narrow, intense lines in their spectrograms, similar to our N-type waves. We observed the latter only for $f=0$, never for $f \neq$ 0 , where only broad structures exist. A question is whether the different geometry of the imposed magnetic field is sufficient to explain these different results. Note also that the differential rotation does not play an important role in the Maryland experiment, since rotation is almost uniform outside the tangent cylinder. To summarize, while some qualitative features of both Couette flow experiments are similar, a quantitative agreement is not achieved.

The present observations might be related to temporal variations in the Earth's magnetic field that have been analyzed recently. Constructing time-longitude diagrams of the magnetic field at the core surface - after removing steady and axisymmetric components - for different latitudes and over the past 400 years, Finlay \& Jackson (2003) pinpoint westwards moving structures with preferred azimuthal wavenumbers, depending on latitude and ranging from $m=8$ at $20^{\circ} \mathrm{N}$ to $m=5$ at the equator and to lower wavenumbers at $40^{\circ} \mathrm{S}$. On the other hand, our observations have no obvious counterparts in numerical simulations of the Earth's dynamo. This can be attributed to a pervasive influence of viscous forces in presently available numerical solutions. It has indeed been argued recently that decreasing the Prandtl number - and thus the magnetic Prandtl number makes non-axisymmetric and fluctuating components of the magnetic field predominant (Simitev \& Busse, 2005).

In conclusion, several types of magneto-inertial waves have been evidenced in our rapidly rotating spherical Couette flow experiment DTS, all retrograde relative to the sodium flow. They constitute the major part of the fluctuations which thus appear to be "quantized" around preferential frequencies rather than distribuded continuously. Their main characteristics have been determined (dependence on the forcing, selection rules for their azimuthal number, dispersion relations for each type). Their life time has been found to be consistent with the order of magnitude of the Lundquist number in our experiment. Concerning the triggering mechanism, no definite answer has emerged from our results. A process involving high shear regions due to strong differential rotation seems plausible, since the waves are inhibited for a weak forcing. A clear identification of the nature of waves is not available at the present time. The experimental characteristics of DTS are far from the conditions required for producing pure inertial waves: strong shear, large magnetic field, strong magnetic field gradient leading to strong spatial variations of Lorentz forces. Conversely, conditions for obtaining pure Alfvén waves are not fulfilled, because of the strong rotation. In DTS, all the characteristic times (inertial, diffusive, Alfvénic) are of same order of magnitude, so that the different terms in the wave equations have to be considered all together. The proximity of all these characteristic times is a strong argument for magneto-inertial waves where all the physical ingredients (rotation, shear, magnetic field) are important. Therefore, the nature of the observed waves can be clarified only with numerical investigations, taking into account realistic physical parameters, velocity shear and flow-induced magnetic field. In the prospect of future liquid-metal dynamo experiments, we anticipate that the waves identified in this work 
will be prominent features. Larger experiments with possibly larger self-generated magnetic fields will produce larger Lundquist numbers and the conditions for the existence of magneto-inertial waves will be met. Similarly, future numerical models of the geodynamo with lower magnetic diffusivity should contain this type of waves: they are old theoretical predictions and we now have some experimental evidence of their existence.

Acknowledgments: Jean-Paul Masson and Patrick La Rizza are gratefully acknowledged for their skillful technical assistance and Alexandre Fournier for useful discussions. The DTS project is supported by Fonds National de la Science, Institut National des Sciences de l'Univers, Centre National de la Recherche Scientifique, Région Rhône-Alpes and Université Joseph Fourier.

\section{References}

ACheson, D. J. 1978 On the instability of toroidal magnetic fields and differential rotation in stars. Royal Society of London Philosophical Transactions Series A 289, 459-500.

Acheson, D. J. \& Hide, R. 1973 Hydromagnetics of rotating fluids. Reports of Progress in Physics 36, 159-221.

Alfvén, H. 1942 Existence of electromagnetic-hydrodynamic waves. Nature 150, 405406.

Bloxham, J., Zatman, S. \& Dumberry, M. 2002 The Origin of Geomagnetic Jerks. Nature 420, 65-68.

BRAGinsKY, S. 1970 Torsional magnetohydrodynamic vibrations in the earth's core and variations in day length. Geomagnetism and Aeronomy 10,1-8.

Braginsky, S. I. 1980 Magnetic waves in the core of the earth. II. Geophysical and Astrophysical Fluid Dynamics 14, 189-208.

Cardin, P., Brito, D., Jault, D., Nataf, H.-C. \& Masson, J.-P. 2002 Towards a rapidly rotating liquid sodium dynamo experiment. Magnetohydrodynamics 38, 177 .

Dormy, E. \& Mandea, M. 2005 Tracking geomagnetic impulses at the core mantle boundary. Earth and Planetary Science Letters 237, 300-309.

Finlay, C. C. 2005 Hydromagnetic waves in Earth's core and their influence on geomagnetic secular variation. $\mathrm{PhD}$ thesis, University of Leeds.

FinlaY, C. C. \& JACKSON, A. 2003 Equatorially Dominated Magnetic Field Change at the Surface of Earth's Core. Science 300, 2084-2086.

Gailitis, A., Lielausis, O., Platacis, E., Dement'ev, S., Cifersons, A., GerBeth, G., Gundrum, T., Stefani, F., Christen, M. \& Will, G. 2001 Magnetic field saturation in the riga dynamo experiment. Phys. Rev. Lett. 86, 3024-3027.

Gailitis, A., Lielausis, O., Platacis, E., Gerbeth, G. \& Stefani, F. 2003 The Riga dynamo experiment. Surveys in Geophysics 24, 247-267.

Glatzmaier, G. A. \& Roberts, P. H. 1996 Rotation and Magnetism of Earth's Inner Core. Science 274, 1887-1891.

Gubbins, D. \& Roberts, P. 1987 Magnetohydrodynamics of the Earth's Core. In Geomagnetism (ed. J. A. Jacobs), , vol. 2, pp. 1-184. Academic Press.

HidE, R. 1966 Free hydromagnetic oscillations of the earth's core and the theory of geomagnetic secular variation. Phil. Trans. Roy. Soc. Lond. A 259, 615-647.

Jameson, A. 1964 A demonstration of Alfven waves. Part 1. Generation of standing waves. Journal of Fluid Mechanics 19, 513-527. 
Jault, D., Gire, C. \& Le Mouel, J. L. 1988 Westward drift, core motions and exchanges of angular momentum between core and mantle. Nature 333, 353-356.

Jault, D. \& LÉgaut, G. 2005 Alfvén waves within the Earth's core. In Fluid Dynamics and Dynamos in Astrophysics and Geophysics (ed. A. M. Soward, C. A. Jones, D. W. Hughes \& N. O. Weiss), pp. 277-+.

Kelley, D. H., Triana, S. A., Zimmerman, D. S., Brawn, B., Lathrop, D. P. \& Martin, D. H. 2006 Driven inertial waves in spherical Couette flow. Chaos 16, $1105-+$.

Kelley, D. H., Triana, S. A., Zimmerman, D. S., Tilgner, A. \& Lathrop, D. P. 2007 Inertial waves driven by differential rotation in a planetary geometry. Geophysical and Astrophysical Fluid Dynamics 101 (5-6), 469-487.

Kitchatinov, L. L. \& Ruediger, G. 1997 Global magnetic shear instability in spherical geometry. Mon. Not. R. Astron. Soc. 286, 757-764.

Lehnert, B. 1954 a Magneto-Hydrodynamic Waves in Liquid Sodium. Physical Review 94, 815-824.

LehneRt, B. 1954b Magnetohydrodynamic Waves Under the Action of the Coriolis Force. Astrophysical Journal 119, 647.

LuNDQUisT, S. 1949 Experimental Investigations of Magneto-Hydrodynamic Waves. Physical Review 76, 1805-1809.

Malkus, W. V. R. 1967 Hydromagnetic planetary waves. Journal of Fluid Mechanics 28, 793-802.

Monchaux, R., Berhanu, M., Bourgoin, M., Moulin, M., Odier, P., Pinton, J.-F., Volk, R., Fauve, S., Mordant, N., Pétrélis, F., Chiffaudel, A., Daviaud, F., Dubrulle, B., Gasquet, C., Marié, L. \& Ravelet, F. 2007 Generation of a Magnetic Field by Dynamo Action in a Turbulent Flow of Liquid Sodium. Physical Review Letters 98 (4), 044502-+.

Motz, R. O. 1966 Alfvén Wave Generation in a Spherical System. Phys. Fluids 9, $411-412$.

Mueller, U., Stieglitz, R. \& Horanyi, S. 2004 A two-scale hydromagnetic dynamo experiment. J. Fluid Mech. 498, 31-71.

Nataf, H.-C., Alboussière, T., Brito, D., Cardin, P., Gagnière, N., Jault, D., Masson, J.-P. \& Schmitt, D. 2006 Experimental study of super-rotation in a magnetostrophic spherical Couette flow. Geophysical and Astrophysical Fluid Dynamics 100, 281-298.

Nataf, H.-C., Alboussière, T., Brito, D., Cardin, P., Gagnière, N., Jault, D. \& SchmitT, D. 2008 Rotating spherical Couette flow in a dipolar magnetic field: an experimental study of the mean axisymmetric flow. Physics of the Earth and Planetary Interiors (under revision).

Reese, D., Rincon, F. \& Rieutord, M. 2004 Oscillations of magnetic stars. II. Axisymmetric toroidal and non-axisymmetric shear Alfvén modes in a spherical shell. $A \& A$ 427, 279-292.

Roberts, P. 1967 An introduction to Magnetohydrodynamics. Elsevier.

Roberts, P. H. \& Soward, A. M. 1972 Magnetohydrodynamics of the Earth's Core. Annual Review of Fluid Mechanics 4, 117-154.

Rüdiger, G., Hollerbach, R., Schultz, M. \& Elstner, D. 2007 Destabilization of hydrodynamically stable rotation laws by azimuthal magnetic fields. Mon. Not. R. Astron. Soc. 377, 1481-1487.

Simitev, R. \& Busse, F. H. 2005 Prandtl-number dependence of convection-driven dynamos in rotating spherical fluid shells. Journal of Fluid Mechanics 532, 365-388.

Sisan, D. R., Mujica, N., Tillotson, W. A., Huang, Y.-M., Dorland, W., 
Hassam, A. B., Antonsen, T. M. \& Lathrop, D. P. 2004 Experimental Observation and Characterization of the Magnetorotational Instability. Physical Review Letters 93 (11), 114502-+.

Sisan, D. R., Shew, W. L. \& Lathrop, D. P. 2003 Lorentz force effects in magnetoturbulence. Physics of the Earth and Planetary Interiors 135, 137-159.

Spruit, H. C. 1999 Differential rotation and magnetic fields in stellar interiors. Astronomy and Astrophysics 349, 189-202.

Stefani, F., Gundrum, T., Gerbeth, G., Rüdiger, G., Schultz, M., Szklarski, J. \& Hollerbach, R. 2006 Experimental Evidence for Magnetorotational Instability in a Taylor-Couette Flow under the Influence of a Helical Magnetic Field. Physical Review Letters 97 (18), 184502-+.

Stieglitz, R. \& Mueller, U. 2001 Experimental demonstration of a homogeneous two-scale dynamo. Phys. Fluids 13, 561-564.

Zhang, K., Liao, X. \& Schubert, G. 2003 Nonaxisymmetric Instabilities of a Toroidal Magnetic Field in a Rotating Sphere. The Astrophysical Journal 585, 1124-1137. 